\title{
Living antennas on communication satellites
}

\author{
Lumholt, Michael
}

Published in:

I E E E Potentials

Link to article, DOI:

10.1109/MP.2003.1197875

Publication date:

2003

Document Version

Publisher's PDF, also known as Version of record

Link back to DTU Orbit

Citation (APA):

Lumholt, M. (2003). Living antennas on communication satellites. I E E E Potentials, 22(2), 13 - 15.

https://doi.org/10.1109/MP.2003.1197875

\section{General rights}

Copyright and moral rights for the publications made accessible in the public portal are retained by the authors and/or other copyright owners and it is a condition of accessing publications that users recognise and abide by the legal requirements associated with these rights.

- Users may download and print one copy of any publication from the public portal for the purpose of private study or research.

- You may not further distribute the material or use it for any profit-making activity or commercial gain

- You may freely distribute the URL identifying the publication in the public portal

If you believe that this document breaches copyright please contact us providing details, and we will remove access to the work immediately and investigate your claim. 
nternational crises, such as the Cold War, the Gulf War and the War on Terrorism, change not only the political landscape, but also the global pattern of communication. The demand for communications over "hot spots" leaps dramatically during their moment in the sun. In turn, high demands are placed on the antenna designers for communication satellites whose coverage can be revamped.

When an international crisis occurs, there is an instant demand for an extraordinarily high level of communications from the crisis region. Crowds of reporters move to the region to make newscasts, which are transmitted to the news station via satellite links. The extraordinarily high demand for communication from the crisis region to the surrounding world often surpasses the capacity of the satellites available for transmitting the signals. The major stations aggravate this shortage by keeping a communication link busy for several hours before using it. They do it simply to ensure that they are able to broadcast live during their prime time.

In the long run, crises also change the global pattern of communication. Old enemies become new allies and new international markets open. In the course of only a few years, an increased demand will arise for effective communication between countries and continents that previously did not communicate much. Just think of the improved relations between the West and the former Soviet republics and Eastern Europe after the Cold War.

The communications problems occur because the satellites are optimized to cover specific geographic areas, and these areas cannot be altered once the satellites are in Earth orbit. An effective solution to the problem is to equip communication satellites with "living" antennas that can adjust their radiation coverage areas according to the new demands. The development of living antennas is, therefore, among the focus areas identified and supported by the European Space Agency, ESA.

\section{The existing satellites}

The existing communication satellites direct their radiated energy into specified geographical areas with potentially high volumes of traffic. These coverage areas usually have an irregular shape, such as the one defined by the contours of a country or a continent as seen from the satellite's position. An example of a European coverage area is shown in Fig. 1. A contoured radiation like this is achieved by using advanced antennas that are optimized to the specific coverage area.

Communication satellites have one major disadvantage once built and launched into orbit: They are out of reach for repairs and changes. With a production time of typically two years and an expected lifetime of about 15

\section{LIVING ANTENNAS}

\section{ON COMMUNICATION}

\section{SATELLITES}

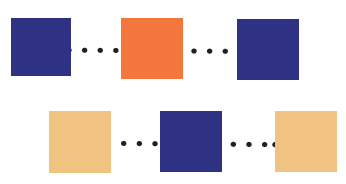

Michael Lumholt

years after launch, the decisions made in the specification phase constrain the use of the satellite for many years.

\section{Making changes}

With existing technology, the coverage area cannot be changed significantly once the satellite is positioned in its geosynchronous orbit. So why not just fly up to the satellites and change the antennas? Manned flights, e.g. with the space shuttle, never take place further

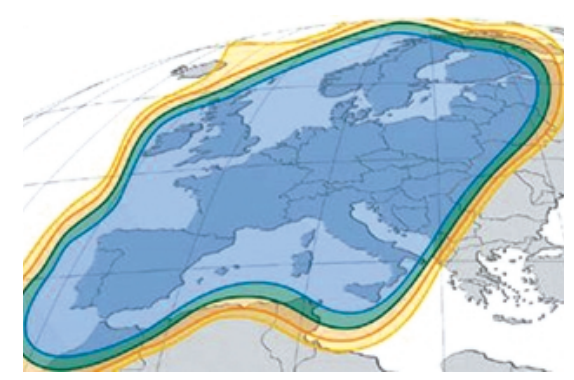

Fig. 1 A European coverage from the Astra 2C satellite Illustration: SES ASTRA. away than 1,000 kilometers. But, within this distance, it is possible to repair a satellite as was done in 1992 (Fig. 2).

An Intelsat VI communication satellite was stranded in low Earth orbit because of a malfunction in the launch vehicle separation system. The crew of the space shuttle EndeavorEndeavour maneuvered to the satellite, captured it, installed a new motor and released the satellite again. When Endeavour was at a safe distance from the satellite, the new motor was fired to bring the satellite to the geosynchronous orbit originally intended.

But, with the existing manned space vehicles, such a repair is impossible once the satellite reaches a geosynchronous orbit of 36,000 kilometers above the surface of the Earth. The extra mass, complexity and energy required to launch the vehicle into geosynchronous orbit, maneuver to the satellite for repair, and finally return the astronauts to Earth is too expensive. It would be similar to what is required to go to the moon. Thus, this method is not feasible for changing the coverage area of a communication satellite.

Unmanned flights are also not feasible. Even though the space vehicle does not need to return to Earth, huge developments within robot technology must be carried out before it is possible to make in-orbit replacement of the antennas on the satellite. The cost of such a

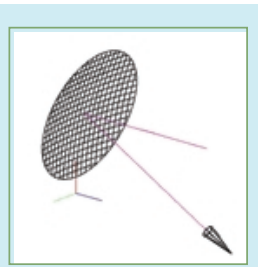
Box 1
A short intro to the
reflector antenna

In its most simple form, the reflector antenna consists of a feed horn for radiating (or receiving) electromagnetic energy and a curved reflecting surface, the reflector. It concentrates and directs the energy towards a confined region in space called the antenna main beam. The larger the reflector is, the more concentrated the radiated energy becomes, and the higher gain has the antenna. The shape of the reflector surface is usually a part of a paraboloid, in which case a circular main beam is obtained.-ML 


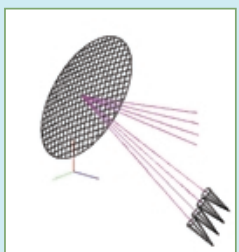

Box 2

Multi-beam

antennas

The multi-beam antenna consists of a reflector surface-usually with parabolic shape-illuminated by a number of feed horns. Each horn produces a circular main beam slightly displaced on the Earth from the other beams. By carefully selecting the excitation of the individual feed horns in amplitude as well as in phase, the superposition of all the individual beams produces the specified contoured beam. The individual excitation of the feed horn is obtained by feeding the input signal through a so-called beam-forming network.-ML

mission would be far too high compared to just producing and launching an entirely new communication satellite.

The logical option is to develop communication satellites carrying living antennas, which can change their radiation in-orbit. In the antenna community, these living antennas are referred to as reconfigurable antennas or adaptive antennas.

\section{Today's antennas}

Most antennas used for communication satellites today are so-called reflector antennas. The principle of this type of antenna is summarized in Box 1 . Usually, reflectors with a parabolic shaped surface are used. When such a reflector is illuminated by a single feed horn, a circular main beam is obtained.

A contoured coverage area can be obtained from two variations of the reflector antenna with a parabolic shaped surface: the multi-beam antenna and the shaped-reflector antenna. The multi-beam antenna is the most widely used of the two. Usually, it consists of a parabolic reflector illuminated by a large number of feed horns. Each single feed horn is excited through a beamforming network in such a way that the main beam of the radiated pattern has the specified shape. This type of antenna is described in more detail in Box 2.

The shaped-reflector antenna is a more recent type of antenna. Instead of illuminating a parabolic reflector with several feed horns in a beam-forming network, a single feed horn illuminates a reflector having a surface which is deformed in a controlled way (Box 3).

For For a relatively simple coverage area, e.g. the coverage of Western Europe as shown in Fig. 1, the shapedreflector antenna has a number of advantages compared to the multibeam antenna: It has less electrical loss, it is lighter and cheaper to produce. Furthermore, the beam-forming network in the multi-beam antenna may require tuning. This is a process that can take several months and may increase the time required to deliver the ordered satellite.

For complicated coverage areas, the multi-beam antenna has an advantage, though. Since the designer has more degrees of freedom, he or she can achieve a more optimized design.

\section{The reconfigurable multi-beam antenna}

The principle of making the multibeam antenna reconfigurable is simple. The excitation of all feed horns must be adjustable while the satellite is in orbit. This can be achieved by controlling the so-called switches and phase shifters that

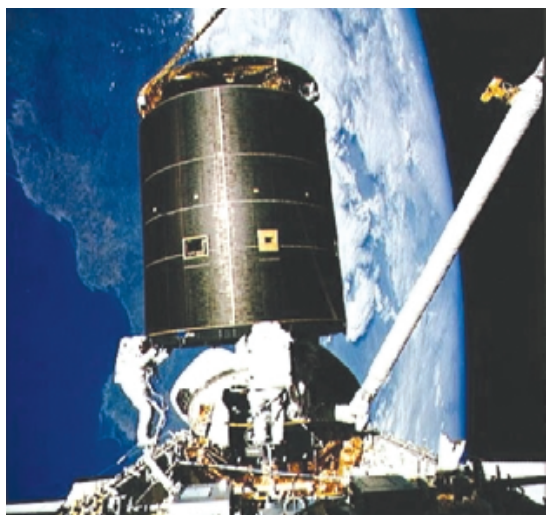

Fig. 2 Astronauts capture the Intelsat VI satellite stranded in low earth orbit. Photo: NASA

are part of the beam-forming network.

However, the realization of an adjustable beam-forming network for use in space is not straightforward. At present, research is being conducted to develop the advanced beam-forming network in a technology that has sufficiently low mass and sufficiently low electrical loss.

Some of the existing communication satellites use a simple type of reconfigurable multi-beam antennas. The beamforming network can switch between two or three configurations, each of which produces a different coverage area. This technique has been used for example on the Intelsat VI satellites. Important to note is that these reconfigurable antennas can only switch between coverage areas already decid- ed upon in the design phase. If a need arises outside these areas, the satellite cannot be used. Hence, this type of antenna does not solve the problem of a changed political landscape as mentioned earlier.

\section{The reconfigurable shaped-reflector antenna}

The principle of the reconfigurable shaped-reflector antenna is simple too. The shape of the reflector surface must be adjustable while the satellite is in orbit. This is possible if the surface is made of a flexible material connected to a number of actuators on the back of the reflector antenna. The desired shape of the flexible surface can be achieved by changing the actuator settings.

The practical application of shaped reflector technology has been made possible by two developments: 1) composite materials with extremely low coefficients of thermal distortion, and 2) the availability of sophisticated computer software packages necessary to design and analyze the antenna.

The flexible surfaces create a number of mechanical problems, though, which are currently being investigated. For example, it has to be ensured that the flexible surface with the connected actuators can survive the vibrations inside the rocket during its launch.

\section{A new type of reconfigurable surface}

Reflector antennas with reconfigurable surfaces have been investigated in Europe for some time. They use a reflector surface consisting of a thin metallic tricot mesh, woven in the same way as a lady's stocking. The surface is controlled with actuators at a number of points.

Unfortunately, a surface of this kind develops a logarithmic singularity at the points where the actuators are attached. As a result, it assumes the form of a pillow with buttons. In terms of material science, the singularities occur because the tricot surface has no

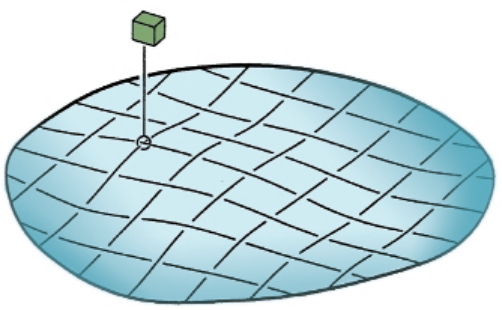

Fig. 3 Engineering sketch of the bending stiff surface with a single actuator. 
bending stiffness. The singularities cause a reduction in the power of the main beam of the antenna and, hence, are undesirable.

An improved functionality could be obtained using a surface with bending stiffness. Therefore, the Danish engineering company TICRA has investigated such surfaces for the European Space Agency, ESA. The study revealed a new type of surface consisting of a mesh of interwoven bendable wires, such as piano wires. At some of the wire connections, the surface is

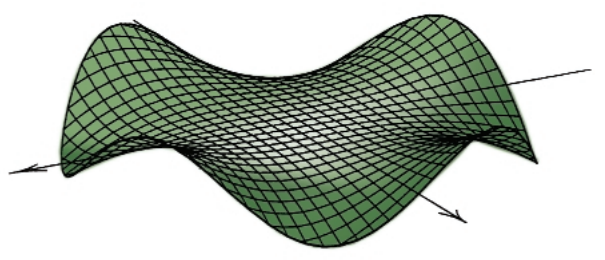

Fig. 4 The surface, when the edge curve, $F$, is given as $F=\sin (4 \theta)$.

connected to actuators. The principle is sketched in Fig. 3, where only a few of the wires and only a single actuator are shown. The surface is bending stiff, which means that it remains smooth under the deformations even at the points connected to the actuators.

Thus, this type of surface is expected to have improved functionality compared to the surfaces with singularities. To verify this, the mathematical model of the surface needed to be implemented in TICRA's software for designing and analyzing shaped-reflector antennas. However, the model of the surface was not available in the literature. Hence, the development of such a model became part of the antenna study. The mathematical problem was solved as a fourth order partial differential equation. The surface has several surprising properties. This may be exemplified considering a surface with a circular edge with the wires oriented along the $\mathrm{x}$ - and $\mathrm{y}$-axis. First, a sinusoidal edge curve, $\mathrm{F}$, is considered defined by $F=\sin (4 \theta)$, where $\theta$ is the angle from the $\mathrm{X}$-axis. The surface in Fig. 4 is obtained, which equals zero at the center, identical to the average value of the edge curve.

Next, the edge curve, $F=\cos (4 \theta)$, is considered. The edge corresponds to a rotation of $22.5^{\circ}$ of the edge in the example just considered (Fig. 4). At first, one would expect a surface that is simply a rotation of $22.5^{\circ}$ compared to the surface in Fig. 4. This is not the case. Instead, a completely different surface is obtained (Fig. 5). The value at the center does not equal zero but rather $-55 / 57$, i.e. almost identical to the minimum value -1 along the edge. In other words, the surface is highly anisotropic, i.e. the surface properties are not the same in all directions.

The behavior of the surface differs for the two edge curves due to the different orientation of the wires relative to the edge. In Fig. 4, the wires are oriented symmetrically at the maxima and minima along the edge. In Fig. 5, on the other hand, the wires are oriented differently at the maxima and minima.

To verify the mathematical model, a scaled model of the reflector surface with a diameter of $12 \mathrm{~cm}$ was manufactured (Fig. 6). The surface wires were made by optical fibers, which possess the desired flexibility. The surface model shows the predicted behavior for the two edges' curves and, thusthus, verifies the mathematical model.

A number of strings can be attached as actuators to the surface at different points. It is surprising to see how complicated, yet smooth, a surface can be

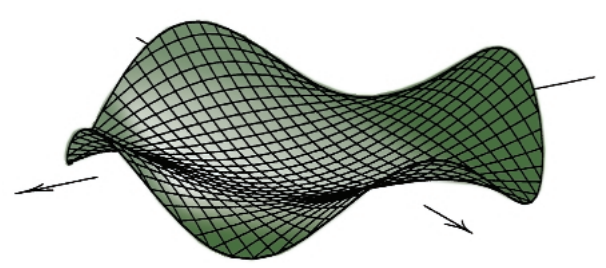

Fig. 5 The surface, when the edge curve, $F$, is given as $F=\cos (4 \theta)$.

obtained using only a hand-full of strings. The surface is indeed highly flexible.

In summary, the study resulted in the development of a surface with the desired bending stiffness. The surface consists of a fabric of interwoven flexible wires, which makes it highly aniso-

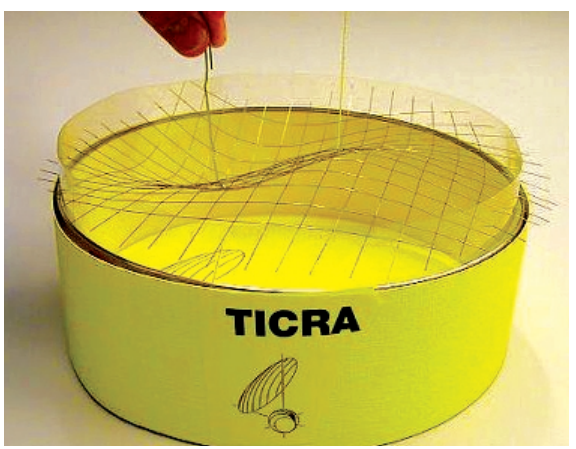

Fig. 6 A scaled model

tropic. However, the anisotropic behavior does not constitute a problem as the

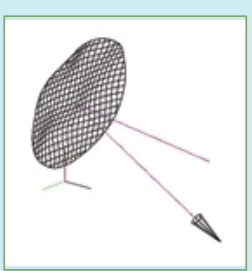

Box 3

Shaped-reflector antennas

In the shapedreflector antenna, the reflector is illuminated by a single feed horn. The contoured main beam is obtained by adjusting the shape of the reflector surface. Popularly speaking, one introduces a number of bumps in the reflector surface. These bumps degrade the radiated circular main beam in such a controlled way that the desired coverage area is obtained.-ML

mathematical model for the surface behavior has been derived. The model has a significant advantage when implemented in shaped-reflector design software: it allows the direct optimization of the reflector-actuator settings. This is precisely the data required for the satellite operators in order to change the coverage area of the satellite.

The achievements possible using this type of reconfigurable surface are still unknown. It is in the early development phase. We hope the surface will be used for reconfigurable antennas on future communications satellites in Earth's orbit. These antennas will then be able to fulfill one of the major desires of the satellite operators: The ability to fit the satellite rapidly to the changing communication needs.

\section{Read more about it}

- P.J.B. Clarricoats et al., Recent developments in reconfigurable reflectors, IEEE AP-S Symposium, Dallas, Texas, May 7-11, 1990.

- N.C. Albertsen et al., Mathematical treatment of an adjustable surface formed by a fabric of interwoven flexible wires, Math. Engng. Ind., vol. 6, no. 2, pp. 115-132, 1997.

\section{About the author}

Michael Lumholt, Ph.D. from the Technical University of Denmark, works for TICRA (www.ticra.com), an engineering company that provides consultancy within antennas and electromagnetic theory. The company further develops industry-standard antenna software for satellite manufacturers worldwide. He has been a member of IEEE since 1993. In the Danish Astronautical Society, he has been a member of the board since 1991 and president since 2002 . 